another through the weak force. Weak charges can therefore be determined by comparing the behaviour of left- and right-handed versions of particles.

To extract the proton's weak charge, the $Q_{\text {weak }}$ Collaboration fired beams of electrons that had a particular handedness at a proton target. They measured an asymmetry that describes the difference in the probability that right- and left-handed electrons are scattered from the proton (Fig. 1). The authors found an asymmetry of $-226.5 \pm 9.3$ parts per billion, where the minus sign indicates that left-handed electrons are more likely to be scattered than their righthanded counterparts. To put the magnitude of this asymmetry in perspective: if parity symmetry were violated for the height of mountains, Mount Everest and its mirror-image twin would differ in height by a mere 2 millimetres, and this difference would have been measured to a precision of $\pm 80 \mu \mathrm{m}$.

The authors' result has a much higher precision than all previous experiments that studied parity violation by scattering electrons from a nuclear target. The E158 experiment at the SLAC National Accelerator Laboratory in Menlo Park, California, had a comparable precision, but measured the weak charge of the electron rather than that of the proton ${ }^{2}$. The $Q_{\text {weak }}$ Collaboration used its measured asymmetry to determine that the proton's weak charge is $0.0719 \pm 0.0045$, which is in excellent agreement with the value predicted by the standard model of particle physics ${ }^{3}$. For comparison, in the convention used by the authors, the proton's electric charge is +1 .

One might question why physicists want to measure the proton's weak charge to such high precision. The short answer: to test the limits of our knowledge. At a basic level, physicists seek to discover if, and at what length scale, current theories fail to explain observational data. Such a failure could imply the existence of a fifth fundamental force - a previously undiscovered type of interaction that has a role at energies higher than have been explored so far.

The measurement reported by the $Q_{\text {weak }}$ Collaboration shows that such interactions, if they exist, would reveal themselves at particle energies beyond several $\mathrm{TeV}(1 \mathrm{TeV}$ is $10^{12}$ electronvolts). For comparison, the energies released in nuclear-fission reactors, in which nuclei split into two or more fragments, are at the level of $10^{6} \mathrm{eV}$ per particle. The authors' lower limit for the energy scale of new physics is comparable to, and complements, that set by experiments at the Large Hadron Collider at CERN, Europe's particlephysics laboratory near Geneva, Switzerland ${ }^{4,5}$. This is remarkable, given that the energy of the authors' electron beams is thousands of times lower than that of the Large Hadron Collider's proton beams.

More than a century ago it was demonstrated that electric charge comes in discrete chunks ${ }^{6}$, which provided a bridge between classical

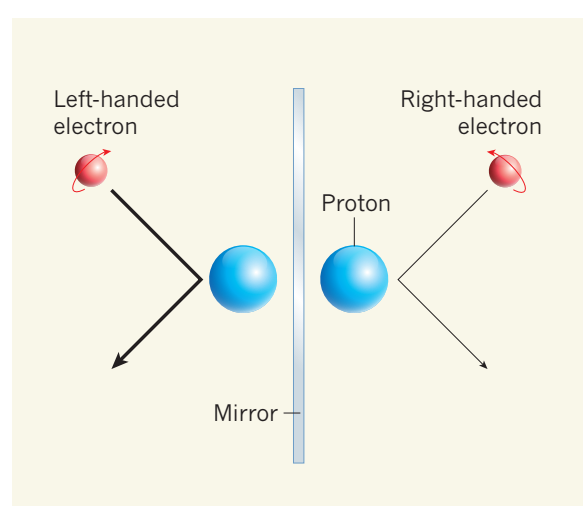

Figure 1 | Parity violation in electron-proton scattering. Electrons can be either left- or righthanded, depending on the direction in which they spin (red arrows) relative to their motion (black arrows). The Jefferson Lab $Q_{\text {weak }}$ Collaboration ${ }^{1}$ reports that left-handed electrons are slightly more likely to be scattered from a proton target than are their right-handed counterparts. This result violates a principle known as parity symmetry because the two scattering processes are mirror images of each other. The authors used the measured asymmetry to determine the value of a fundamental physical quantity known as the weak charge of the proton to an unprecedented precision. electromagnetism and modern quantum mechanics. By measuring quantities - from physical constants to particle properties such as the proton's weak charge - at increasingly higher precision, new interactions and particles could be discovered that require current theories to be revised. Such lofty pursuits can be maintained only if research is carried out by multiple groups across generations, as opposed to single groups in isolated instances. One day, the knowledge acquired might lead to a breakthrough. In the meantime, it will make our world a better one by providing a deeper understanding of the physical laws of nature.

Xiaochao Zheng is in the Department of Physics, University of Virginia, Charlottesville, Virginia 22904, USA.

e-mail:xz5y@virginia.edu

\footnotetext{
1. The Jefferson Lab $Q_{\text {weak }}$ Collaboration. Nature 557, 207-211 (2018).

2. SLAC E158 Collaboration. Phys. Rev. Lett. 95, 081601 (2005)

3. Patrignani, C. et al. (Particle Data Group). Chin. Phys. C 40, 100001 (2016).

4. ATLAS Collaboration. Eur. Phys. J. C 74, 3134 (2014)

5. CMS Collaboration. Phys. Lett. B 746, 79-99 (2015).

6. Millikan, R. A. Phys. Rev. 2, 109-143 (1913).
}

\title{
Connections that control defence strategy
}

When presented with a threat, most mice freeze or hide, but a few respond more aggressively. The brain circuits underlying this behavioural choice have now been unpicked. SEE ARTICLE P.183

\section{DAYU LIN}

$\mathrm{M}$ y nine-year-old daughter got a pair of mice for Christmas. To acclimatize her new pets to her presence, she put her hands into their cage. One of the mice ran straight to its shelter and hid; the other stayed in a corner, held its tail up against the glass wall and rattled it rapidly. Tail rattling is a common response to stress across species (most famously in snakes ${ }^{1}$ ), and is considered a warning to intruders ${ }^{1,2}$. But why do some mice hide, and others threaten? What brain mechanisms determine which defensive strategy to deploy in the face of potential danger? On page 183, Salay et al. ${ }^{3}$ reveal a brain region that could be responsible for this decision in mice.

Salay et al. first surveyed the brain regions that are activated by threatening cues. They placed mice in a test arena, then presented them with an overhead looming stimulus - an expanding dark-coloured circle, to mimic an approaching predator. When the mice detected the looming cues, they either froze or quickly ran towards a shelter under which they could hide from the stimulus. Occasionally, mice rattled their tails once they had reached the safety of the shelter.

To identify the neurons that determine these behavioural responses, the researchers analysed the protein c-Fos, which is expressed rapidly in neurons after they have been highly active. Many brain regions show consistently high levels of c-Fos in response to looming, and one caught the authors' attention - the ventral midline thalamus (vMT). The vMT is interesting in that it is not a part of the eye-tobrain visual pathway or a motor pathway, as might be expected for neurons involved in processing visual stimuli such as the looming cues in this study. Instead, it receives diffuse inputs from limbic areas and the midbrain (regions that support emotion, motivational behaviours and bodily responses), and projects heavily to higher cognitive areas, such as the prefrontal cortex ${ }^{4}$. Thus, this region is well suited to signalling the internal state of the animal and guiding its defence strategies. 

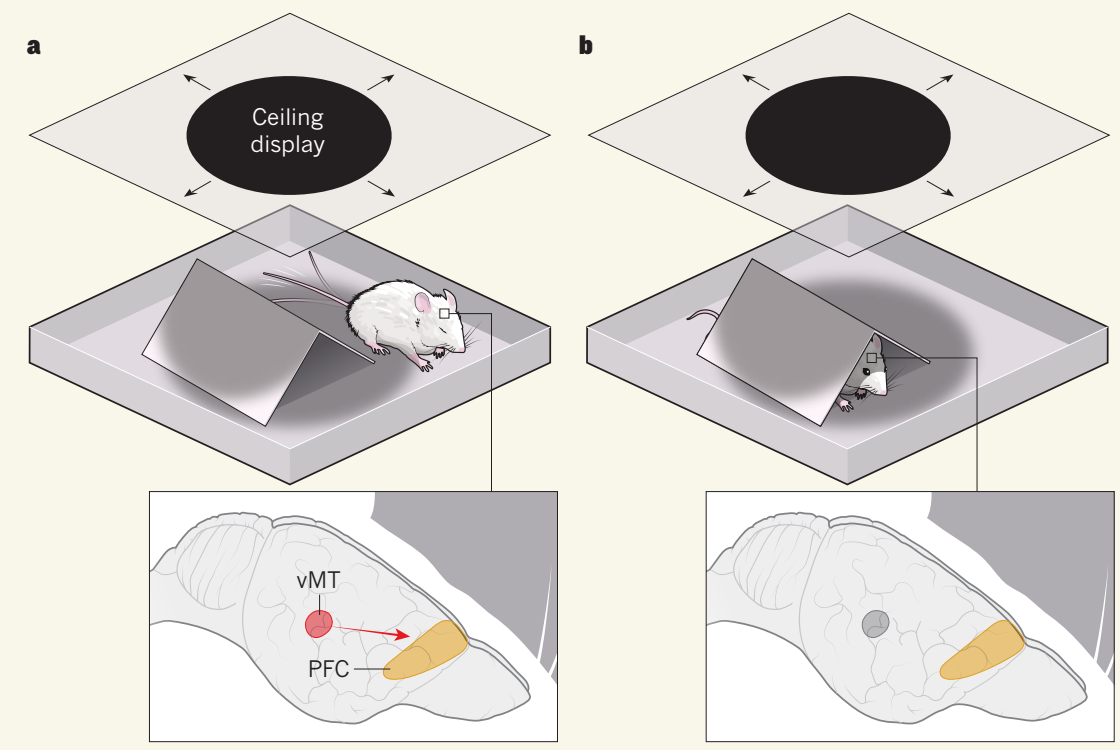

Figure 1 | To recede or to challenge. Salay et al. ${ }^{3}$ report that a region of the brain known as the ventral midline thalamus (vMT) controls how a mouse responds to a threatening visual stimulus, such as an expanding dark circle in a ceiling display that looms overhead. a, The authors provide evidence that signalling from the vMT to the prefrontal cortex (PFC) promotes an active defence strategy, such as making an aggressive tail-rattling motion. $\mathbf{b}$, By contrast, when levels of vMT activation are lower, mice respond to the looming stimulus by hiding.

In vivo recordings of neuronal activity revealed that vMT cells are most active when the animals are responding actively to looming stimuli by running and tail rattling. Salay et al. therefore asked whether vMT activity is sufficient to guide an animal's defence strategy. The authors injected the vMT with a viral construct carrying a receptor protein that, when activated, excites neurons. The receptor is activated on binding to a small ligand, which the authors administered systemically weeks after injection of the virus. Strikingly, when Salay and colleagues artificially activated vMT cells, the mice no longer hid from the looming stimuli, but instead rattled their tails frequently and ran around the open space. Conversely, when vMT cells were suppressed using a similar approach, the animals spent more time freezing and never rattled their tails in response to looming stimuli.

To investigate how vMT activation affects responses to looming in more detail, the authors genetically modified cells such that they could be activated by a laser, giving millisecond-level control of cell activity. They found that activating vMT cells either in tandem with or 30 seconds before the presentation of looming stimuli promoted the same active defence behaviours, such as tail rattling. This behavioural shift could outlast the stimulation itself, which makes it likely that activating the vMT causes a shift in internal state, instead of eliciting acute motor actions. Consistent with this hypothesis, the researchers showed that vMT activation induces strong and longlasting autonomic responses indicative of increased arousal, such as pupil dilation, but does not induce changes in motor activity in the absence of looming stimuli.

Next, Salay et al. investigated which projections from the vMT are relevant for guiding defence behaviours. They found that distinct regions of the vMT project to the prefrontal cortex and basolateral amygdala, both of which are implicated in many complex functions, including fear and anxiety. The authors again used a viral technique, this time to manipulate specific vMT outputs, and found that activating vMT projections to the prefrontal cortex pathway promoted an active threat-coping strategy (tail rattling) and increased arousal (Fig. 1). By contrast, activating the vMT-basolateral amygdala projection favoured a different behaviour, freezing. Taken together, the authors conclude that distinct vMT outputs control opposing threat-coping strategies.

Escaping and hiding are basic survival instincts when an animal is faced with a powerful predator. However, from time to time, prey animals need to stand their ground. For instance, adult ground squirrels protect pups in a burrow fiercely - after detecting a nearby snake, the squirrel flags its tail and aggressively harasses the snake by biting and kicking dirt towards it. As a result, the snake typically abandons its hunting effort and moves away. When a weak animal challenges a stronger predator in this way, the animal might be considered 'brave. Does it stand to reason, then, that the vMT drives a 'courageous' state?

Answers to this question remain unclear,

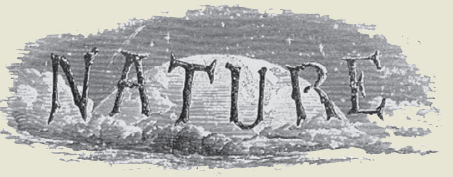

50 Years Ago

Britain's first heart transplant operation, performed last week at the National Heart Hospital, has been received with the usual gush of publicity which accompanies such events. But less acclaim has been given to the liver transplantation operation carried out at Addenbrooke's Hospital, Cambridge, on May 2, although this is technically by far the trickier of the two operations ... Several attempts at liver transplants have been made in the United States, but met with little success until June of last year. Since then advances in immunosuppression and organ storage have enabled the livers of deceased persons to be maintained in a form acceptable to the recipient's body for several hours ... The heart surgery team at the National Heart Hospital says that the heart transplant operation is the first of a series of operations leading to a combined heart-lung transplant before the end of the year.

From Nature 11 May 1968

100 Years Ago

The Gas Traction Committee, appointed in November last to consider the employment of coal-gas as a substitute for petrol and petroleum products in motors, its general safety, and conditions for use, has issued an interim report ... This deals chiefly with the present use of gas, mainly at low pressures, in suitable fabric bags; the work is to be continued to cover its use under higher compressions when the necessary appliances and plant are obtainable. The Committee is satisfied that gas can be efficiently, safely, and promptly substituted for motor spirit (only two minor accidents have been reported). Two hundred and fifty cubic feet are considered equivalent to one gallon of petrol, so that gas at $4 s$. per 1000 cub. $\mathrm{ft}$. is equivalent in cost to petrol at $1 s$. per gallon.

From Nature 9 May 1918 
because a direct assessment of courage is not possible in non-human species. Intriguingly, however, the internal state elicited by vMT activation seems to be perceived as positive, because the mice prefer to stay in a chamber in which they can receive the vMT stimulation, given a choice. Future studies that examine the correlation between vMT activity and courage in humans will help us to further understand the emotional state encoded by the vMT.

It is also still unclear whether tail rattling in mice can effectively deter a predator in the wild. But this behaviour did successfully stop my daughter from bothering her pets.
Dayu Lin is at the Neuroscience Institute, New York University School of Medicine, New York, New York 10016, USA.

e-mail:dayu.lin@nyumc.org

1. Greene, H. W. J. Herpetol. 7, 143-161 (1973).

2. Haber, S. B. \& Simmel, E. C. Bull. Psychonom. Soc. 7, 84-86 (1976).

3. Salay, L. D., Ishiko, N. \& Huberman, A. D. Nature 557, 183-189 (2018)

4. Van der Werf, Y. D., Witter, M. P. \& Groenewegen, H. J. Brain Res. Rev. 39, 107-140 (2002).

5. Clark, R. W. Behav. Ecol. Sociobiol. 59, 258-261 (2005).

This article was published online on 2 May 2018.

\section{A closer look at human telomerase}

The telomerase enzyme performs a crucial role by maintaining protective caps of repetitive DNA sequences at the ends of chromosomes. A structure of human telomerase casts fresh light on its architecture. SEE ARTICLE P.190

\section{MICHAEL D. STONE}

$\mathrm{T}$ The chromosomes of eukaryotic organisms, which include plants, animals and fungi, are capped by protective structures called telomeres ${ }^{1}$. In certain types of proliferative cell, these structures are maintained by the telomerase enzyme. Inherited genetic mutations that compromise telomerase function cause disorders characterized by deterioration of proliferative tissues ${ }^{2}$, whereas increased expression of telomerase supports unbridled cell growth in most human cancers ${ }^{3}$. Efforts to develop telomerase-based therapeutics have been hampered by an incomplete understanding of the structure and organization of the telomerase complex. On page 190, Nguyen et al. ${ }^{4}$ report a structure of human telomerase bound to DNA, providing an unprecedented view of how the enzyme complex is organized and establishing a framework for drug discovery.

Telomerase is a ribonucleoprotein (RNP) enzyme - part RNA, part protein. To retain normal function, the enzyme must contain at least a telomerase reverse transcriptase (TERT) protein subunit and a long, non-coding telomerase RNA (TR). Telomerase complexes also usually include several species-specific proteins that are important for regulating RNP assem$b^{5} y^{5}$. The enzyme maintains telomeres by catalysing the addition of simple DNA-sequence repeats onto the ends of linear chromosomes. The catalytic TERT subunit uses a small portion of TR as a template for these repeats.

Vertebrate telomerases also belong to the family of box H/ACA RNP complexes, which generally modify essential cellular RNAs such as transfer RNAs, ribosomal RNA and spliceosomal RNA ${ }^{6}$. Curiously, despite possessing the RNA and protein components of H/ACA RNPs, telomerase does not exhibit the RNAmodifying activity typically associated with this class of complex. Nevertheless, mutations in those components impair telomere maintenance and cause diseases related to telomerase deficiency ${ }^{2}$.

Human telomerase has low abundance in cells, and attempts to reconstitute it from its purified components have been thwarted by protein insolubility and the low efficiency of RNP assembly. This challenge can be overcome by expressing TERT and TR in cultured human cells that naturally express large quantities of the other telomerase proteins needed for RNP assembly ${ }^{7}$. This approach was used to reconstitute human telomerase and produce the first low-resolution structure ${ }^{8}$ of the enzyme using electron microscopy (EM). However, telomerase complexes made in this way can contain different subunits and have varying structures, both of which limit the resolution of EM structures.

Nguyen et al. report an improved purification procedure that yields substantially moreactive and more homogeneously assembled telomerase. Armed with this highly purified enzyme, the authors used cryo-electron microscopy (cryoEM) to reconstruct the structure of human telomerase at subnanometre resolution. This resolution is three times better than that of the previously reported structure, and allowed the authors to reliably work out how the various telomerase components 